International Journal of Methods in Psychiatric Research

Int. J. Methods Psychiatr. Res. 15(2): 95-103 (2006)

Published online in Wiley InterScience

(www.interscience.wiley.com) DOI: 10.1002/mpr.183

\title{
The use of propensity score methods in psychiatric research
}

\author{
TYLER VANDERWEELE ${ }^{1}$
}

1 Harvard School of Public Health, Boston, Massachusetts, USA

\begin{abstract}
The use of propensity score methods has become increasing widespread in public health, in medicine and in many other settings involving research with observational studies. The methods provide a useful and straightforward way of simultaneously controlling for many variables that potentially confound the relationship between a specific outcome and the treatment or exposure under study. The methods assist the researcher in avoiding certain dangers in more traditional regression modelling and, in the case of binary outcomes, allow for the control of a greater number of potential confounders. The use of propensity score techniques has been fairly limited in psychiatry, largely to studies of health care delivery and use. An introduction to propensity score methods is given and a series of examples illustrating their use in psychiatric research is presented. Copyright (C) 2006 John Wiley Eु Sons, Ltd.
\end{abstract}

Key words: propensity score, causal inference, stratification, matching, weighting

\section{Introduction}

When researchers are unable to conduct randomized clinical trials, data from observational settings are used to make inferences about the effects of different treatments. However, in observational studies, individuals receiving different treatments are likely to differ from one another in a number of other respects. Data must be collected on those variables that are thought to confound the relationship between the treatment and outcome. Adjustment is made for these confounding variables. There are a variety of statistical methods that allow for multivariate control of several confounding variables. One such method, which is very useful and, for reasons outlined below, arguably superior to other techniques, is the use of the propensity score.

Although propensity score methods have seen increasingly widespread use, these techniques have not been used very extensively within the psychiatric literature. This article will provide researchers with an introduction to propensity score methods, demonstrate the potential usefulness of these methods in psychiatric research and thereby promote further application of these techniques. In the first section of the article, a description of the propensity score and the theory underlying its use is presented. In the second section, a brief outline is given of each of eight psychiatric research studies employing propensity score methods. In the third section, issues concerning the implementation of propensity score methods are considered, with examples drawn from the eight psychiatric studies. The discussion is summarized in the conclusion.

\section{The propensity score}

For the purpose of making causal inferences, ideally, we would like to know what would have happened to each individual under each possible treatment scenario. A counterfactual outcome is what would have happened to an individual had he or she received a treatment other than the one that was in fact given. In reality, we are only able to observe the outcome for that individual under the particular treatment that was in fact received. We are unable to observe what the outcome would have been had that individual received a different treatment (crossover designs represent a partial 
exception to this; these designs, however, suffer from potential carryover effects and various other limitations). Individuals receiving different treatments will probably differ on a number of other variables as well, so data are collected on as many variables as possible that are thought to affect the relationship between treatment and outcome. We call variables that potentially affect relationships between treatment and outcome confounding variables. A confounding variable may be conceived of as a variable that is affected by neither the treatment nor the outcome but rather itself affects both the treatment and the outcome such that adjustment for the variable reduces the bias in estimating the causal effect. If all confounding variables have been identified then, within strata of these confounding variables, individuals will differ only with respect to the treatment that had been received. In such cases, formally, we say that treatment assignment is independent of the counterfactual outcomes conditional of the confounding variables. Individuals who have the same value on the confounding variables can be compared to obtain unbiased estimates of the treatment effect. Of course other sources of bias, for example selection bias or measurement error, may also be present (Maclure and Schneeweiss, 2001). But in the absence of these other sources of bias, the process of conditioning on confounding variables allows conclusions to be drawn about the causal effects of treatments; propensity score methods are one approach to go about estimating these causal effects. For further discussion of the principles of causal inference the reader may consult one of several general review articles on this topic (Kaufman and Poole, 2000; Little and Rubin, 2000; Maldonado and Greenland, 2002).

The propensity score is the probability of a particular individual being assigned treatment conditional on some group of observed variables. Suppose each subject has some observed covariates $X$ and an indicator of treatment $\mathrm{Z}$ with $\mathrm{Z}=1$ if the individual is treated and $Z=0$ otherwise. Then the propensity score $e(X)$ is given by $\operatorname{Prob}(Z=1 \mid X)$. The propensity score has certain theoretical properties that make it particularly useful in trying to control for multiple confounders. Rosenbaum and Rubin (1983a) have shown that if it suffices to adjust for $\mathrm{X}$ to estimate the effect of treatment then it also suffices to adjust instead only for the propensity score $\mathrm{e}(\mathrm{X})$; that is, if the counterfactual outcomes are independent of treatment conditional on $\mathrm{X}$ then the counterfactual outcomes are also independent of treatment conditional on $\mathrm{e}(\mathrm{X})$. The advantage of adjusting for the propensity score $\mathrm{e}(\mathrm{X})$ rather than $\mathrm{X}$ itself is that $e(X)$ is a single variable and stratification on a single variable is relatively straightforward. The set X may contain dozens of variables, making stratification and other methods of adjustment difficult if not impossible.

Note that if there are any unmeasured confounders (variables that affect the relation between treatment and outcome that are not included in X) then adjustment based on the propensity score is not sufficient for calculating valid estimates of the treatment effect. But in such cases, adjustments for X itself will not suffice either. This is simply a limitation of all observational studies as compared with randomized experiments. Furthermore, as discussed above, whenever adjustment for $\mathrm{X}$ suffices (i.e. whenever there are no unmeasured confounders), adjustment for e(X) also suffices. When a researcher does suspect the presence of unmeasured confounders it is possible under additional assumptions to gauge the extent to which this might alter the estimate of the treatment effect by means of sensitivity analysis techniques (Rosenbaum and Rubin, 1983b; Brumback et al., 2004; Greenland, 2005; MacLehose et al., 2005).

The result that if it suffices to adjust for $\mathrm{X}$ to estimate the effect of treatment then it also suffices to adjust only for the propensity score $\mathrm{e}(\mathrm{X})$ is in some ways surprising. It suggests that we can replace a multivariate adjustment or stratification procedure by a univariate one. Why is it that this property holds? Some intuition can be given by describing another theoretical property of the propensity score, also demonstrated by Rosenbaum and Rubin (1983a): if a stratum is homogeneous in the propensity score then the treated subjects within that stratum and the control subjects within that stratum will have the same distribution of the observed covariates X. In other words, if we stratify on the propensity score then, within each stratum, all the observed covariates tend to be balanced between the treatment and control groups. In effect, as far as concerns the observed covariates, it is as though we have conducted a randomized experiment within each stratum. Unlike a randomized experiment, however, stratification by use of a propensity score balances only those covariates that have been observed. It does not balance those covariates that have not been observed.

Various implementation issues are critical for the use of propensity score methods, including the estimation 
of propensity scores, balance amongst covariates, questions of stratification, matching and weighting and consideration of various extensions. Before taking up these issues, a brief description of eight psychiatric research studies employing propensity score methods is given.

\section{Overview of psychiatric studies using propensity score methods}

Brief descriptions of eight psychiatric research studies that have made use of the propensity score in conducting data analysis are presented below. Searches were conducted using the PubMed database to identify articles published up through 30 September 2003; only articles published in English were considered. Many of the articles identified by the search criteria given below made use of the word 'propensity' but not in relation to 'propensity scores' but rather in the sense of 'propensity to,' 'propensity for,' or 'propensity towards.' The searches also identified many articles making use of propensity score methods but outside the field of psychiatric research. Only those articles that in fact employed propensity score methods for psychiatric studies were included in the article's discussion.

The criteria 'Propensity Score Psychiatric' gave 11 results and two relevant articles (Clark and Rich, 2003; Mojtabai and Zivin, 2003). The criteria 'Propensity Scores Psychiatric' gave 18 results and two additional relevant articles (Rosenheck et al., 2000; Evans and Boothroyd, 2002). The criteria 'Propensity Score Mental' and 'Propensity Scores Mental' gave 12 and 19 results respectively but no additional relevant articles. The more general criteria 'Propensity Score' gave 222 results and one additional relevant article (Sosin, 2002). The criteria 'Propensity Scores' gave 175 results but no additional relevant articles. The criteria 'Propensity Matching' gave 48 results but no additional relevant articles. The criteria 'Regression Matching' gave 624 results and two additional relevant articles (Reinisch et al., 1995; Phillips et al., 2000). One of these final articles (Phillips et al., 2000) made reference to another study (Phillips et al., 1997), which employed propensity score techniques but was not identified by the search criteria above. Thus, in total, eight psychiatric research studies employing propensity score methods were identified and are described below.

These studies illustrate the usefulness of propensity score methods and draw attention to the diversity of potential settings within which such methods can be employed. It should be noted that the research design and analysis in each of these articles have certain limitations and unavoidable weaknesses including issues concerning generalizability, dropouts and unmeasured confounders; the authors discuss many of these. However, it is not within the scope of this article to provide a general critique of the research design and methods in these articles but merely, in each case, to highlight the role of the propensity score in the analysis of the data. The studies themselves are described below; the specific use these studies made of the propensity score is described in the following section on implementation.

Clark and Rich (2003) used propensity score methods to examine the effect of two types of service programmes in ameliorating homelessness among individuals with severe mental illness. Two types of programmes were compared; first, a comprehensive housing programme in which consumers received guaranteed access to housing, housing support services and case management and, second, a service programme that provided case management only. Adjustment for background characteristics was made by using propensity score stratification.

Phillips et al. (2000) used propensity score methods as one of several techniques to control for bias in a study examining whether the placement of nursing home Alzheimer patients in Alzheimer special care units (SCUs) allowed caregivers to avoid the use of potentially dangerous interventions such as physical restraints and psychotropic medications. There were four outcomes of interest: whether the resident received physical restraints any time in the 7 days preceding the assessment, whether the resident was given psychotropic medication any time in the 7 days preceding the assessment, whether the resident had received both psychotropic medication and physical restraints, or whether the resident had received neither. Propensity score methods were used to form samples matched on background characteristics. In another analysis (Phillips et al., 1997), the same data and a very similar analytic approach were used to examine the effects of residence in an SCU on functional outcomes.

Sosin (2002) examined the effect of case management and housing services on drinking, drug use and homelessness. Comparison was made between three groups: those receiving housing services, those receiving case management only, and a control group. The 
study design employed was that of a randomized experiment. Background characteristics were assessed including the baseline level of the outcome variables and various theoretically derived predictors. In follow-up interviews conducted a year after programme entry, the following outcomes were measured: the days clients reported using alcohol in the 30-day period prior to the interview; the days they reported using drugs in the same period, and the days they reported being homeless in the 60-day period prior to the interview. As is perhaps common with studies of this kind, once the assignment was made, large numbers in each group, especially those not assigned to the housing programme, refused to participate. The author's primary objective was to explore various methods of attempting to correct for the resulting sample selection bias; one of the methods employed was that of propensity score matching.

Reinisch et al. (1995) conducted a study on the effect of in utero exposure to phenobarbital on intelligence in men. Subjects were selected from the largest hospital in Copenhagen. After the application of various exclusion criteria, the exposure group consisted of those who had been exposed in utero to phenobarbital and the control group of those who had not. Propensity score matching and regression techniques were used to adjust for background characteristics in making intelligence comparisons.

Rosenheck et al. (2000) examined the effect of compensated work therapy on post-traumatic stress disorder (PTSD) within Veterans Affairs (VA) inpatient programmes. The control group consisted of veterans who were in specialized VA inpatient programmes for treatment of PTSD but not specifically in compensated work therapy programmes. Seven outcome measures were taken at baseline and at 4 months follow-up including two scales for detection of post-traumatic stress disorder, a violence index, an alcohol problem index, a drug problem index, a medical problem index and an employment index. Propensity score methods were used to control for background characteristics for each of these seven outcomes.

Mojtabai and Zivin (2003) examined the effectiveness and cost-effectiveness of four treatment modalities for substance disorders. Subjects were taken from a random sample of drug treatment facilities. Each treatment facility was classified as one of the following for purposes of comparison: inpatient, residential, outpatient detox/methadone, or outpatient drug free. The outcomes measured at five years after discharge from the facility were abstinence and the presence of any reduction in substance use. Effectiveness and costeffectiveness for each of the four treatment modalities were estimated, adjusted for background covariates by propensity score stratification.

Evans and Boothroyd (2002) compared youth referred to psychiatric emergency services by police with those referred by other sources. The study subjects consisted of children and adolescents referred to psychiatric emergency services in the Bronx. Upon referral, clinical staff completed the Screening Crisis among Children and Adolescents with Reported Emergencies (SCCARE) assessment. The study's outcomes include substance abuse, exhibition of assaultive and destructive behaviour and treatment referral. Various background characteristics from the SCCARE were adjusted for by propensity score matching. It would seem however that, for the purposes of causal inference, propensity score techniques were used incorrectly in this study: outcomes such as substance abuse and exhibition of assaultive and destructive behaviour took place before the police referral (the exposure) and so could not have been caused by it.

\section{Implementation issues}

\section{Estimation of the propensity score}

The theoretical results presented in section one suggest useful and convenient methods for using the propensity score to control for confounding variables. In practice, however, the propensity score is unknown and must be estimated. This is most typically done by means of a logistic regression; the treatment indicator is used as the dependent variable in a logistic regression with the observed pretreatment variables $\mathrm{X}$ as the independent variables; the estimated probabilities are used as the propensity scores. Surprisingly, it turns out that under certain circumstances the estimated propensity scores can perform just as well as or even better than the true propensity scores (Rosenbaum, 1984; Joffe and Rosenbaum, 1999). An estimated propensity score cannot distinguish between systematic imbalance in the covariates and imbalance in the covariates that is due to chance. Stratifying on an estimated propensity score therefore tends to eliminate both types of imbalance, whereas adjustment for the true propensity score, even if it were known, would only remove systematic bias. A more formal explanation of this phenomenon 
depends on the notion of an ancillary statistic (Robins et al., 1992). The disadvantage of needing to use estimated propensity scores is that the propensity score must be modelled and this runs the danger of misspecification. Although logistic regression models have most frequently been used to estimate the propensity score, other models are also conceivable such as a probit model or the use of discriminant analysis (Little and Rubin, 2000). Amongst the studies described above, Sosin (2002) estimated the propensity scores by means of a probit model (similar to a logistic regression model but with the inverse normal function as a link instead of the logit function); the other studies outlined above used logistic regression to estimate the propensity scores.

\section{Stratification on the propensity score}

Three major approaches to use the propensity score in multivariate adjustment have been proposed: stratification, matching and weighting. Sometimes regression is employed in conjunction with these techniques. Adjustment by stratification on the propensity score is the most straightforward and perhaps most widespread method of adjustment and therefore will be covered in greatest detail here. Matching and weighting will also be discussed.

When stratifying on the estimated propensity scores a decision needs to be made as to the number of strata to be used. Exact stratification cannot be used because typically each subject will have a different estimated propensity score. When stratifying on the propensity score it has become common practice to use five strata. Cochran (1968) proves various theoretical results showing that if there are a reasonable number of both treatment and control subjects in each strata then five or six strata are usually sufficient to remove at least $90 \%$ of the bias in the crude estimates obtained without adjustment. Amongst the studies referenced above that employed propensity score stratification, Rosenheck et al. (2000), Mojtabai and Zivin (2003) and Evans and Boothroyd (2002) all used five propensity score strata while Clark and Rich (2003) used three strata due to the limited size of the data set. Estimated propensity score quintiles, of both groups combined or in the treated or control group alone, are most often used in practice as boundaries for each strata. Hullsiek and Louis (2002) have contributed some theoretical work as to the best way to choose strata boundaries. After estimating the propensity scores and fixing the strata boundaries, the distribution of propensity scores of the treatment and control groups can be compared to ensure that there is sufficient overlap in the distributions and a sufficient number of both treatment and control subjects in each strata. If a particular stratum has a small number of either treatment or control subjects, estimates of the treatment effect for that stratum will be imprecise; furthermore, if there is not sufficient overlap in the propensity score distributions, it may be that subjects with particular covariate patterns have no meaningful counterfactuals (are either always in the treatment group or always in the control group) and that comparison for such individuals is not possible.

Balance in covariates between treatment and control groups within each stratum can then be assessed using t-tests. If a substantially greater number of significant differences is found between covariates than would be expected by chance then the propensity score model should be reformulated so as to include interaction or quadratic terms among the covariates. Covariate balance between treatment and control groups within each stratum can then be checked again. If it is not possible to obtain fairly good covariate balance within strata then it may be that covariate distributions do not overlap sufficiently to allow stratification to adjust for these covariates. If the covariates are relatively balanced within each stratum it is then reasonable to compute the difference in outcome between treatment and control groups within each stratum and then to obtain an overall estimate of the treatment effect by averaging over the within-strata treatment-control outcome differences weighted by the number of subjects in each stratum. If the numbers of treated within each stratum are used as weights, this will give an estimate of the average treatment effect on the treated. If the numbers of control subjects within each stratum are used as weights, this will give an estimate of the average treatment effect for the control subjects. If the numbers of subjects in each stratum from both groups combined are used as weights, this will give an estimate of the average treatment effect on the entire sample.

To illustrate this approach we describe the steps used in the creation of the propensity score model in the research of Mojtabai and Zivin (2003) on comparing the effectiveness of four treatment modalities for substance disorders. For each two-way comparison of treatment modalities, five strata were formed using the quintiles of the propensity scores as cutoffs and the 
distributions of covariates across treatment modalities were compared to assess whether stratification on the propensity score had achieved covariate balance within strata. For those covariates for which statistically significant differences remained, the interaction terms of that variable with all other variables were entered into the logistic model, the propensity scores were reestimated and the assessment procedure was repeated until no significant differences between the two treatments remained in any of the strata. For measures of effectiveness, odds ratios were computed in each strata for abstinence at 5 years and reduction in substance use at 5 years. Results of the analysis indicated very few differences in effectiveness among the four treatment modalities.

In addition to the estimation of the overall effectiveness of various treatments, stratification can sometimes allow a researcher to identify strata in which the treatment effect is more pronounced. In the study on the effect of housing programmes on homelessness amongst the mentally ill (Clark and Rich, 2003), the estimated propensity scores from the logistic regression were divided to form three strata. Because those receiving only case management had more psychiatric symptoms and greater substance abuse, the authors referred to the three 'tertiles' as 'low-, medium- and highimpairment subgroups'. The authors found that in the low- and medium-impairment subgroups, participants in the programme in which only case management was provided did just as well on housing outcomes as those in the programme providing comprehensive housing services. Among the high-impairment participants, however, the participants in the comprehensive housing programme showed noticeably greater gains than those in the programme providing only case management. Interestingly, their findings imply that those who could benefit most from comprehensive housing are in fact more likely to receive only case management. The authors concluded that intervention programmes to ameliorate homelessness among individuals with severe mental illness ought to be tailored according to an individual's level of impairment. Costs can be controlled by assigning patients with low- or medium-psychiatric impairment to programmes that provide case management only. Effectiveness can be improved by ensuring that patients with high levels of psychiatric impairment are placed in programmes that provide comprehensive housing assistance.
Matching on the propensity score

Another approach that is commonly taken in research employing propensity scores is the use of matching rather than stratification. Several approaches to matching may be discerned in the literature. In the psychiatric literature reviewed above, two distinct matching approaches recur and these two approaches will both be described here. The first involves selecting, for each treated subject, one or more controls by taking the controls with propensity scores closest to that of the treated subject in question. The treated subjects along with the matched controls then form the 'analytic sample'. Phillips et al. (2000) and Sosin (2002) both use this approach. The second approach that is found in the articles considered here involves the formation of propensity score strata as described above and then the random selection of control subjects within each strata so as to maintain a constant $\mathrm{n}: 1$ matching ratio among all strata. Rosenheck et al. (2000) and Evans and Boothroyd (2002) both use this second approach; Evans and Boothroyd (2002) with a 3:1 matching ratio and Rosenheck et al. (2000) with a 6:1 matching ratio. This second approach may be desirable if one is attempting to examine whether the effect of treatment varies over strata. More complex matching strategies are also possible (Rosenbaum and Rubin, 1985) as is illustrated by the research of Reinisch et al. (1995). One advantage of matching on the propensity score over stratification is that, after matching has been performed and an 'analytic sample' has been produced, one can proceed with crude estimation, such as computation of means, without worrying about how to combine estimates of treatment effects across strata. In the case of random selection of matched controls for each treated subject (provided the same matching ratio $\mathrm{n}: 1$ is used in each strata if selection is done by strata), the differences in overall means will provide a valid estimate of the average treatment effect on the treated subjects. The disadvantage of these matching techniques as compared with stratification is that by discarding unmatched subjects, information is lost and estimates may be less precise. Furthermore, these matching approaches require a sufficiently larger group of control subjects than treated subjects for otherwise various treated subjects will be discarded and information will again be lost.

To illustrate the use of matching an example is provided from the research of Phillips et al. (2000). In the study on the effect of residence in an Alzheimer SCU 
on avoiding the use of physical restraints and psychotropic medications (Phillips et al., 2000), the authors chose to use a matching technique in which a comparison group was formed by using those residents in a facility with an SCU but not in the SCU itself and those residents in a facility with no SCU whose propensity scores were most similar to the residents of the SCU. With this matching accomplished, three odds ratios were computed: the odds of receiving only physical restraints to no special intervention; the odds of receiving only psychotropic medication to no special intervention; and the odds of receiving both restraints and medication compared to no special intervention. The authors concluded that SCU residents were restrained at rates that were not significantly different from rates observed in either residents in a facility with no SCU or residents in a facility with an SCU but not in the SCU itself. However, they also concluded that SCU residents were more likely to receive psychotropic medication. They use these results to suggest that there is no clear superiority of SCUs over traditional units in their processes of care.

\section{Weighting by the propensity score}

A third adjustment approach making use of the propensity score is that of weighting. If study subjects are weighted according to the inverse of the probability of receiving the treatment they in fact received conditional on the covariates (Rosenbaum, 1987; Robins et al., 1992) - the reciprocal of the propensity score for the treated group and the reciprocal of 1 minus the propensity score for the control group - then this suffices to yield consistent estimates of the treatment effect without further confounding adjustment, provided that there are no unmeasured confounders. Adjustment by weighting has certain advantages over other approaches making use of the propensity score. Decisions relating to the number of strata and the strata boundaries are unnecessary and the residual bias due to not being able to stratify exactly on the propensity score is asymptotically eliminated. The disadvantage of weighting by the propensity score is that if there are treated subjects with propensity scores close to zero or control subjects with propensity scores close to one, weighting by the inverse of these scores can be very unstable, resulting in estimates with a very large variance. Because of these various advantages and disadvantages, the relative effectiveness of matching and stratification versus weighting remains controversial.
Advantages of propensity score stratification, matching and weighting

As noted in the introduction, there are various statistical methods that allow for multivariate control of several confounding variables. In many cases these methods will give similar results. For example, in the study on the effect of residence in an Alzheimer SCU on avoiding the use of physical restraints and psychotropic medications (Phillips et al., 2000), in addition to propensity score matching, alternative methods of multivariate control were also employed including the use of a multinomial (or polytomous) logistic model and also the use of restriction of the sample on which analysis was conducted; results from the various analyses were then compared. In all cases, the conclusions were very comparable, demonstrating that the results were not an artefact of the modelling technique.

Although various methods of multivariate control will often yield comparable results, adjustment by means of propensity score stratification, matching or weighting has several advantages over certain traditional methods of adjustment such as regression. First, although the use of propensity score methods requires modelling the relationship between the treatment and confounding variables, propensity score methods do not impose any functional form on the relation between outcome and the covariates within each treatment group whereas regression models do. Linear regression forces the relation between outcome and the covariates to be additive; adjustment by propensity score methods do not. Second, standard methods of analysis such as linear regression and logistic regression can be deceptive because they provide no warning about when their use is or is not warranted (Rubin, 1997); these methods make use of linearity assumptions and simply extrapolate. They provide estimates even when there is little or no overlap in the covariate distribution between the treatment and control groups - when the treatment and control groups are not sufficiently comparable in a set of baseline characteristics to make inference about the effect of treatment. Nothing in the standard output of regression software will draw attention to this fact. On the other hand, with propensity score methods, if, within strata, the treatment and control groups do not adequately overlap on various confounding variables, the researcher will see this immediately. Propensity score methods make the assumptions of analysis more assessable and transparent. Finally, simulations (Cepeda et al., 2003) suggest that in the case of a binary outcome, 
when the outcome is comparatively rare (seven or fewer events per confounding variable), estimates from propensity score methods have much better empirical properties than logistic regression estimates - less bias, greater robustness, and less variance.

\section{Further extensions}

These basic propensity score techniques have been extended in a number of further directions; brief mention of some of these is given below.

The various stratification, matching and weighting strategies can be easily extended to situations in which more than two treatments are being compared. When the various treatment groups consist of different dose levels of the same treatment, one approach is to fit an ordinal logistic regression and use the linear predictor (excluding the intercept terms) for the purpose of matching and stratification in order to achieve balance. See the work of Rosenbaum (2002) and others (Lu et al., 2001) for more details. This approach, however, requires certain stronger modelling assumptions. Furthermore, Rubin (1997) points out the danger of drawing conclusions without ensuring that there is sufficient overlap among all two-way comparisons. An alternative approach, which can also be employed even in settings in which there is no clear ordering among treatment groups, is that of computing propensity scores for each possible pair of treatments and using the methods above to make pair-wise comparisons. This was the method employed by the studies of Phillips et al. (2000) and Mojtabai and Zivin (2003).

Little and Rubin (2000) argue that there is evidence from simulations and actual applications that analysis strategies combining propensity score methods and regression techniques can sometimes be superior to either strategy alone. Propensity score stratification and matching leave residual bias as the matches will generally be imperfect and exact stratification is impossible; some of this residual bias can be eliminated using regression to adjust for differences in propensity scores amongst treated and control subjects. The studies of both Reinisch et al. (1995) and Rosenheck et al. (2000) used a propensity score matching technique followed by further statistical modelling to make comparisons.

A number of other extensions are worth noting. A generalization of the propensity score weighting technique described above to settings with time-varying exposures and time-varying confounders is given by Robins et al. (2000). Sosin (2002) used propensity scores as one of a number of methods to correct for sample selection bias. In a particularly innovative use of propensity scores, Mojtabai and Zivin (2003) conducted a full cost-effectiveness analysis for four treatment modalities while adjusting for background characteristics.

\section{Conclusion}

Propensity score methods can be useful in a wide range of contexts. These include the use of propensity scores in stratification and matching in order to control for variables that confound the relation between treatment and outcome; their use to address selection bias arising from refusal or dropout in randomized experiments; the use of propensity score stratification and matching as a preliminary stage before proceeding with further modelling; and even their use in cost-effectiveness analysis to ensure comparability of subjects receiving different treatment modalities. Clearly, many of these settings occur frequently within psychiatric research. The application of propensity score methods in these ways - and perhaps a number of others - can be a useful approach in attempting to protect study results against bias.

With these methods for causal inference becoming increasingly accessible to researchers, their application will broaden with time. For the purposes of intervention, it is ultimately causal conclusions that interest the researcher. The more careful thought that is required in addressing issues of causality and the greater effort that is spent in attempting to control for biases arising from confounding will undoubtedly pay off in securing greater validity in study conclusions.

\section{References}

Brumback BA, Hernán MA, Haneuse SJPA, Robins JM. Sensitivity analyses for unmeasured confounding assuming a marginal structural model for repeated measures. Stat Med 2004; 23: 749-67.

Cepeda MS, Boston R, Farrar JT, Strom BL. Comparison of logistic regression versus propensity score when the number of events is low and there are multiple confounders. Am J Epidemiol 2003; 158: 280-7.

Clark C, Rich AR. Outcomes of homeless adults with mental illness in a housing program and in case management only. Psychiatr Serv 2003; 54: 78-83.

Cochran WG. The effectiveness of adjustment by subclassification in removing bias in observational studies. Biometrics 1968; 24: 295-313.

Evans ME, Boothroyd RA. A comparison of youth referred to psychiatric emergency services: police versus other sources. J Am Acad Psychiatry Law 2002; 30: 74-80. 
Greenland S. Multiple-bias modelling for the analysis of observational data. JR Stat Soc A 2005; 168: 267-306.

Hullsiek KH, Louis TA. Propensity score modeling strategies for the causal analysis of observational data. Biostatistics 2002; 3: 179-93.

Joffe MM, Rosenbaum PR. Invited commentary: propensity scores. Am J Epidemiol 1999; 150: 327-33.

Kaufman JS, Poole C. Looking back on 'causal thinking in the health sciences'. Annu Rev Public Health 2000; 21: 101-19.

Little RJ, Rubin DB. Causal effects in clinical and epidemiological studies via potential outcomes: concepts and analytical approaches. Annu Rev of Public Health 2000; 21: $121-45$.

Lu B, Zanutto E, Hornik R, Rosenbaum PR. Matching with doses in an observational study of a media campaign against drug abuse. J Am Stat Assoc 2001; 96: 1245-53.

MacLehose RF, Kaufman S, Kaufman JS, Poole C. Bounding causal effects under uncontrolled confounding using counterfactuals. Epidemiology 2005; 16: 548-55.

Maclure M, Schneeweiss S. Causation of bias: the episcope. Epidemiology 2001; 12: 114-22.

Maldonado G, Greenland S. Estimating causal effects. Int J Epidemiol 2002; 31: 422-9.

Mojtabai R, Zivin JG. Effectiveness and cost-effectiveness of four treatment modalities for substance disorders: a propensity score analysis. Health Serv Res 2003; 38: 233-59.

Phillips CD, Sloane PD, Hawes C, Koch G, Han J, Spry K, Dunteman G, Williams RL. Effects of residence in Alzheimer disease special care units on functional outcomes. J Am Med Assoc 1997; 278: 1340-4.

Phillips CD, Spry KM, Sloane PD, Hawes C. Use of physical restraints and psychotropic medications in Alzheimer special care units in nursing homes. Am J Public Health 2000; 90: 92-6.

Reinisch J, Sanders S, Mortensen E, Rubin DB. In-utero exposure to phenobarbital and intelligence deficits in adult men. J Am Med Assoc 1995; 274: 1518-25.
Robins JM, Hernan MA, Brumback B. Marginal structural models and causal inference in epidemiology. Epidemiology 2000; 11: 550-60.

Robins JM, Mark SD, Newey WK. Estimating exposure effects by modelling the expectation of exposure conditional on confounders. Biometrics 1992; 48: 479-95.

Rosenbaum PR. Conditional permutation tests and the propensity score in observational studies. J Am Stat Assoc 1984; 79: 565-74.

Rosenbaum PR. Model-based direct adjustment. J Am Stat Assoc 1987; 82: 387-94.

Rosenbaum PR. Observational Studies. Section 10.2.4. New York: Spring-Verlag, 2002.

Rosenbaum PR, Rubin DB. The central role of the propensity score in observational studies for causal effects. Biometrika 1983a; 70: 41-55.

Rosenbaum PR, Rubin DB. Assessing sensitivity to an unobserved binary covariate in an observational study with a binary outcome. J R Stat Soc B 1983b; 45: 212-18.

Rosenbaum PR, Rubin DB. Constructing a control group using multivariate matched sampling methods that incorporate the propensity score. Am Stat 1985; 39: 33-8.

Rosenheck R, Stolar M, Fontana A. Outcomes monitoring and the testing of new psychiatric treatments: work therapy in the treatment of chronic post-traumatic stress disorder. Health Serv Res 2000; 35: 133-51.

Rubin DB. Estimating causal effects from large data sets using propensity scores. Ann Intern Med 1997; 127: 757-63.

Sosin MR. Outcomes and sample selection: the case of a homelessness and substance abuse intervention. $\mathrm{Br} \mathrm{J}$ Math Stat Psychol 2002; 55: 63-91.

Correspondence: Tyler VanderWeele, Harvard School of Public Health, Department of Biostatistics, 677 Huntington Avenue, Boston MA 02115, USA.

Telephone: (+1) 617-697-3250.

Email: tvanderw@hsph.harvard.edu 